\title{
Interlaboratory proficiency testing of tensile and flexural testing for cellulose based transformed board material
}

\author{
B. Aydemir and S. Fank ${ }^{\star}$ \\ TUBITAK UME, Force Group Laboratory, Gebze, Kocaeli, Turkey
}

Received: 5 May 2012 / Accepted: 12 June 2012

\begin{abstract}
Optimum safety and reliability can be achieved by coordinating an interlaboratory proficiency testing among different laboratories. Testing laboratories need to verify their test procedures and testing capability for reliable results. In this study, equivalent samples are prepared by cellulose based transformed board material used in transformers as electrical isolation material. Randomly selected test samples having specified dimensions are sent to the participant laboratories. Then they are tested and results are analyzed according to previously defined parameters. Each laboratory applied tensile and flexural tests on the samples according to procedures given in IEC 60641-2, 2004 and IEC 60763-2, 2007 standards respectively. Test results are evaluated according to same procedures explained in related standards by the participant laboratories. TUBITAK UME Material Testing Laboratory being the pilot laboratory collected to the all evaluated results including associated measurement uncertainties. Then the collected results evaluated according to ISO 17043, 2010 are reported by each laboratory as a part of the proficiency test report. Z-scores of the participant laboratories are presented.
\end{abstract}

Keywords: Transformed board; proficiency testing; tensile testing; flexural testing

\section{Introduction}

In Turkey, there is an increasing demand for calibration and certification work obliged by the establishment of calibration laboratories. These laboratories should obey the rules of ISO 17025 [1] for their recognition by the customer and increase the quality of their calibration and test services. In order to give traceable service in testing and calibration area, National Accreditation System controls the laboratories in all countries. TURKAK is the responsible for the organization of accreditation system in Turkey.

Testing laboratories need to verify their own test procedures and testing capability. This verification and testing capability of the accredited laboratories can be achieved by coordinating an interlaboratory proficiency testing among different laboratories. An interlaboratory proficiency testing is organized by the TUBITAK UME to see the closeness and equivalency of the test results among the participating laboratories. Test samples were prepared in cellulose-based transformed board material used in transformers as an electrical isolation material. Each laboratory applied the tensile and flexural tests on the test samples according to procedures given in IEC 60641-2, 2004 and IEC 60763-2, 2007 standards respectively $[2,3]$. All participant laboratories have performed the tests using their own test procedures and own test equipments. Their equipments especially material testing

^ Correspondence: sinan.fank@ume.tubitak.gov.tr machines were calibrated by an accredited laboratory in Turkey. Test results were evaluated according to same procedures explained in related standards by the laboratories. The values of measurements and the associated uncertainties were calculated in accordance with international standards and procedures stated by each laboratory as a part of the test report.

In this paper, the main results obtained during the proficiency test are discussed; in particular an analysis was applied to evaluate the differences in the mean values by the different laboratories. The calculated Z-scores of the test results for the participant laboratories are presented and shown in graphs.

\section{Technical protocol}

First of all, the technical protocol was prepared and it was sent to all participant laboratories. It includes the preparation of the test samples, distribution of them to the laboratories, time table for the tests, test reports and test requirements [4].

\subsection{Participant laboratories and timetable}

Four different test laboratories have participated to this proficiency testing in Turkey. All laboratories declared to provide all special requirements of tensile and bending test 
Table 1. The machines used in the measurements and their specifications.

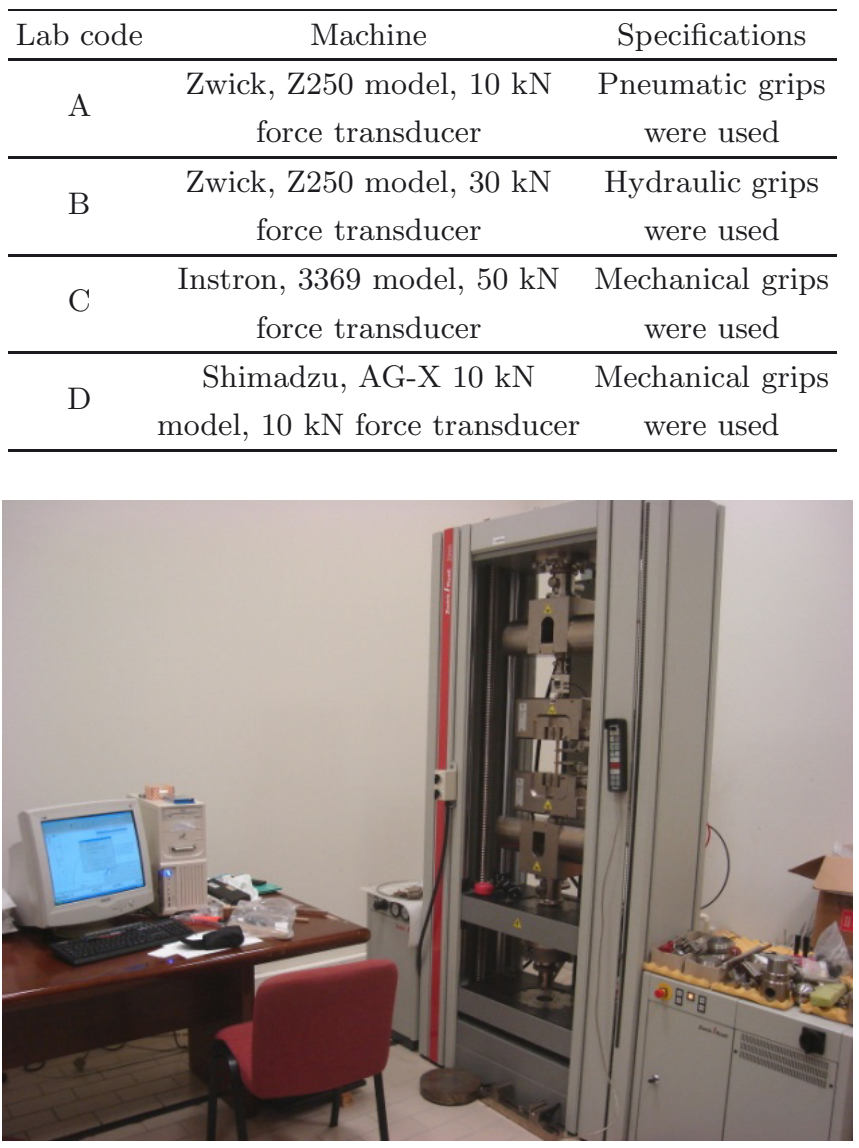

Fig. 1. Material testing machine of TUBITAK UME.

during measurements. Proficiency testing was started on June 27.2011 and was completed on July 01.2011. All measurements were completed according to time schedule declared in technical protocol.

The test machines used in the measurements and their specifications are given in Table 1.

\subsection{Samples and test conditions}

The test samples were prepared according to description given in technical protocol. During the preparation of the test samples in manufacturer, the proficiency test coordinator has observed the all manufacturing and preparation schedules of the test samples. The prepared samples were numbered and packed with utmost care. Each packet was preserved in the same conditions and it was transferred to the laboratories.

Each laboratory tested them on its own test equipments including material testing machine, calliper, and micrometer and so on. The test machine of TUBITAK UME is shown in Figures 1 and 2. All testing equipments of the laboratories were calibrated by an accredited calibration laboratory before starting of the proficiency tests. The test conditions and parameters for the performed

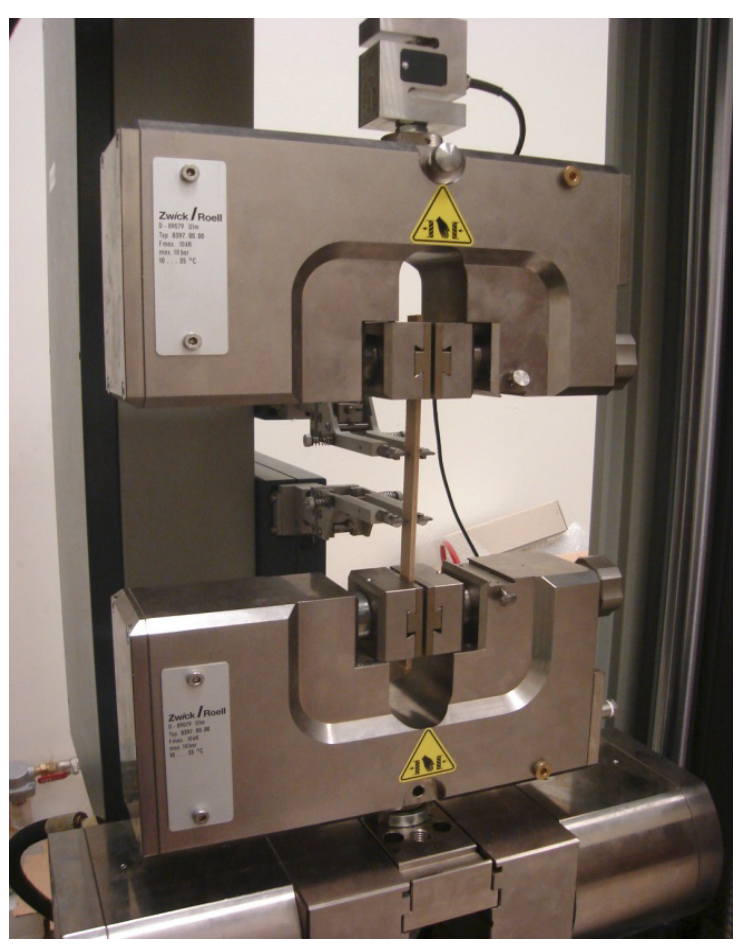

Fig. 2. The photo during tests. Including sample, grips, extensometer and force transducer.

Table 2. Distribution ranges of tensile strengths $\left(\sigma_{t}\right)$ for the participant laboratories.

\begin{tabular}{cccccc}
\hline \multicolumn{5}{c}{$\begin{array}{c}\text { Mean } \\
\text { value }\end{array}$} & \multicolumn{2}{c}{$\begin{array}{c}\text { Standard } \\
\text { deviation }\end{array}$} & value & value & $\begin{array}{c}\text { Distribution } \\
\text { range } \\
\text { (max-min) }\end{array}$ \\
\cline { 2 - 6 } $\mathrm{Lab}$ & \multicolumn{5}{c}{ MPa } \\
\hline $\mathrm{A}$ & 172.81 & 3.96 & 178.69 & 164.15 & 14.54 \\
$\mathrm{~B}$ & 177.89 & 4.04 & 185.41 & 171.57 & 13.84 \\
$\mathrm{C}$ & 173.90 & 4.09 & 179.51 & 165.64 & 13.87 \\
$\mathrm{D}$ & 166.66 & 3.39 & 171.39 & 161.17 & 10.22 \\
\hline
\end{tabular}

tests were conditioned and prepared according to standards of IEC 60641-2 [2] and IEC 60763-2 [3].

\section{Results}

After completing the tests by each laboratory, the test reports were sent to the pilot laboratory which is TUBITAK UME Material Testing Laboratory. TUBITAK UME evaluated all test reports according to ISO 17043 standard [5]. In given figures and tables, each laboratory is represented with a letter as A, B, C or D. The letter "A" represents the test results of TUBITAK UME and it is taken as the reference (pilot) laboratory. The test results and uncertainties of the all laboratories are given in Figures 3-5. Uncertainty calculation of test results of TUBITAK UME is used the training document [6]. The mean values, standard deviations and distribution ranges of the laboratories are given in Tables 2-4. 


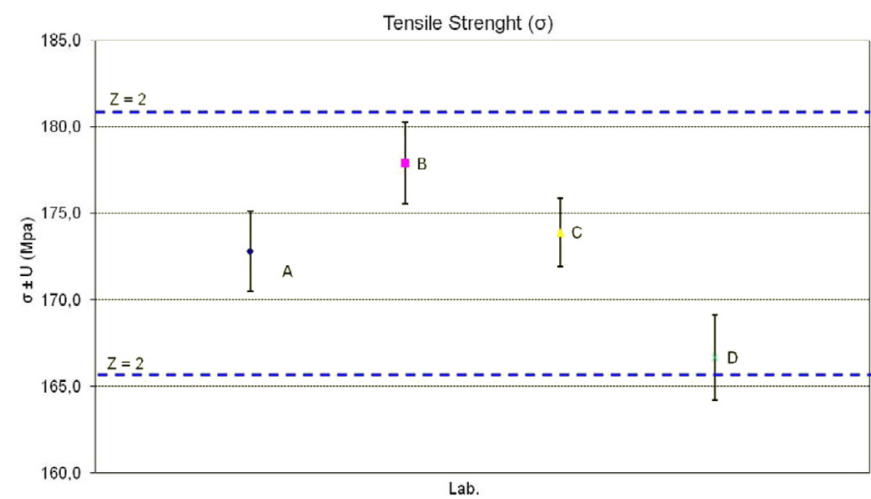

Fig. 3. Tensile strength $\left(\sigma_{t}\right)$ and uncertainty values for the tensile test of the participant laboratories.

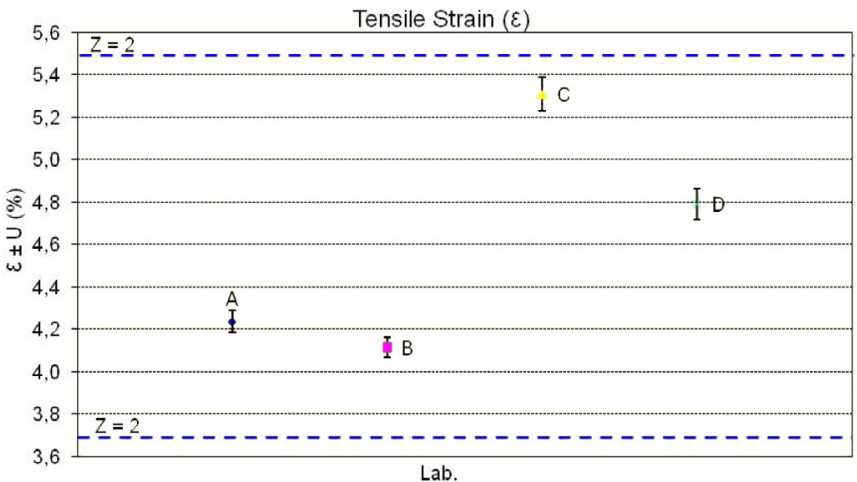

Fig. 4. Tensile strain $(\varepsilon)$ and uncertainty values for tensile test of the participant laboratories.

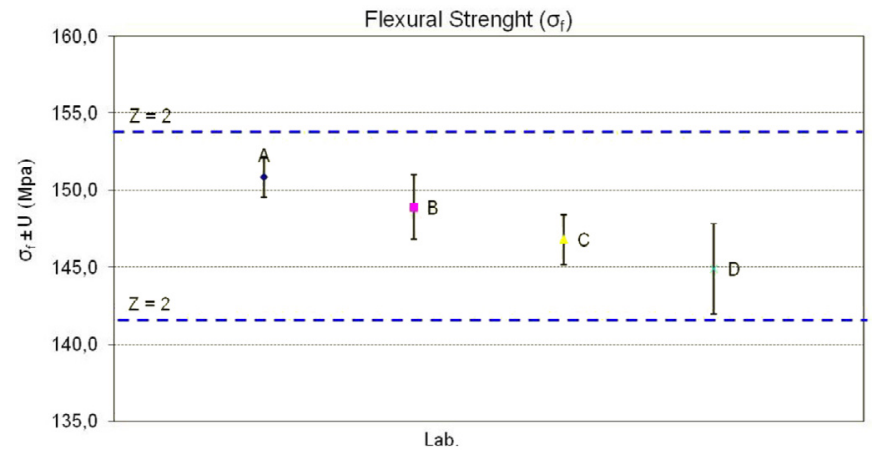

Fig. 5. Flexural strength $\left(\sigma_{f}\right)$ and uncertainty values for flexural testing of the participant laboratories.

The mean tensile strength values were scattered 11.23 $\mathrm{MPa}$ in Table 2. In addition, the average distribution range of each laboratory was determined 13.12 MPa.

In Table 3, the average distribution range of tensile elongation was founded as $0.30 \%$.

The amount of the scattering in the mean flexural strength was founded as $6 \mathrm{MPa}$. At the same time, the average distribution range of each laboratory was observed as 7.69 $\mathrm{MPa}$ in Table 4.

The test reports of the all participant laboratories were sent to the TUBITAK UME then resulted proficiency test report was prepared as well. The test results of the par-
Table 3. Distribution ranges of tensile strains $(\varepsilon)$ for the participant laboratories.

\begin{tabular}{cccccc}
\hline & $\begin{array}{c}\text { Mean } \\
\text { value }\end{array}$ & $\begin{array}{c}\text { Standard } \\
\text { deviation }\end{array}$ & $\begin{array}{c}\text { Maximum } \\
\text { value }\end{array}$ & $\begin{array}{c}\text { Minimum } \\
\text { value }\end{array}$ & $\begin{array}{c}\text { Distribution } \\
\text { range } \\
\text { (max-min) }\end{array}$ \\
\cline { 2 - 6 } $\mathrm{Lab}$ & & \multicolumn{5}{c}{$\%$} & \\
\hline $\mathrm{A}$ & 4.24 & 0.09 & 4.35 & 4.09 & 0.26 \\
$\mathrm{~B}$ & 4.12 & 0.08 & 4.23 & 3.99 & 0.24 \\
$\mathrm{C}$ & 5.31 & 0.13 & 5.47 & 5.11 & 0.35 \\
$\mathrm{D}$ & 4.79 & 0.13 & 4.96 & 4.59 & 0.36 \\
\hline
\end{tabular}

Table 4. Distribution ranges of flexural strengths $\left(\sigma_{f}\right)$ for the participant laboratories.

\begin{tabular}{cccccc}
\hline & $\begin{array}{c}\text { Mean } \\
\text { value }\end{array}$ & $\begin{array}{c}\text { Standard } \\
\text { deviation }\end{array}$ & $\begin{array}{c}\text { Maximum } \\
\text { value }\end{array}$ & $\begin{array}{c}\text { Minimum } \\
\text { value }\end{array}$ & $\begin{array}{c}\text { Distribution } \\
\text { range } \\
\text { Lab }\end{array}$ \\
\cline { 2 - 6 } & \multicolumn{5}{c}{$\mathrm{MPa}-\mathrm{Man})$} \\
\hline $\mathrm{A}$ & 150.84 & 2.24 & 153.37 & 148.06 & 5.31 \\
$\mathrm{~B}$ & 148.88 & 3.53 & 153.95 & 145.81 & 8.14 \\
$\mathrm{C}$ & 146.80 & 1.70 & 148.64 & 144.77 & 3.87 \\
$\mathrm{D}$ & 144.87 & 5.07 & 152.17 & 138.72 & 13.45 \\
\hline
\end{tabular}

Table 5. Calculated Z-score parameters.

\begin{tabular}{cccc}
\hline Lab. & $\sigma_{t}$ & $\varepsilon$ & $\sigma_{f}$ \\
\hline $\mathrm{A}$ & 0.00 & -0.68 & 1.16 \\
$\mathrm{~B}$ & 1.09 & -0.91 & 0.40 \\
$\mathrm{C}$ & 0.23 & 1.27 & -0.41 \\
$\mathrm{D}$ & -1.32 & 0.32 & -1.15 \\
\hline
\end{tabular}

ticipating laboratories were evaluated in according to ISO 17043 standard $[5,7]$.

Z-score is taken as assessment method of the evaluated results. The Z-score is calculated according to below equation:

$$
z=\frac{x-X}{\sigma}
$$

where

- $X:$ the assigned value;

$-x$ : the participant's result;

$-\sigma$ : the standard deviation of test results.

Criteria for performance evaluation should be established after taking into account whether the performance measure involves certain features. The features of Z-scores for performance evaluation are the following:

$-|z| \leqslant 2$ indicates "satisfactory" performance and generates no signal;

$-2<|z|<3$ indicates "questionable" performance and generates a warning signal;

$-|z| \geqslant 3$ indicates "unsatisfactory" performance and generates an action signal.

Tensile strengths $\left(\sigma_{t}\right)$, tensile strains $(\varepsilon)$ and flexural strengths $\left(\sigma_{f}\right)$ for the participated laboratories are given in Table 5 for the calculation of Z-scores $[4,8]$. The evaluations of the Z-scores are also given in Table 6 . 
Table 6. Evaluated Z-scores.

\begin{tabular}{ccccc}
\hline Z-score & $|z| \leqslant 2$ & $2<|z|<3$ & $|z| \geqslant 3$ \\
\cline { 2 - 5 } & $\begin{array}{c}\text { The number } \\
\text { of Lab. }\end{array}$ & $\begin{array}{c}\text { The number } \\
\text { of Lab. }\end{array}$ & $\begin{array}{c}\text { The number } \\
\text { of Lab. }\end{array}$ \\
\hline$\sigma_{t}$ & 4 & 100 & & \\
$\varepsilon$ & 4 & 100 & & \\
$\sigma_{f}$ & 4 & 100 & & \\
\hline
\end{tabular}

\section{Conclusions}

Four (4) different laboratories have participated to the interlaboratory proficiency testing for tensile and flexural tests for cellulose based transformed board material. TUBITAK UME Material testing laboratory has organized to this proficiency testing in 2011.

Initially, technical protocol and test samples were prepared and sent to the participant laboratories. Test measurements carried out in accordance with timetable given in technical protocol. After each laboratory completed all tests, the test reports were sent to TUBITAK UME. All test reports were evaluated by TUBITAK UME according to ISO 17043 standard.

In tensile strength, tensile elongation and flexural strength, the assessment method of Z-score for all laboratories is determined as "success".
In order to verify testing capability of the test laboratories, the proficiency tests are very important for assessment of the tensile and bending test measurement capabilities of all laboratories and it should be repeated periodically.

\section{References}

1. EN ISO 17025, General requirements for the competence of testing and calibration laboratories, 2005

2. IEC 60641-2, Pressboard and presspaper for electrical purposes - Part 2: Methods of tests - 2nd edition, 2004

3. IEC 60763-2, Specification for laminated pressboard - Part 2: Methods of test, edition 2.0, 2007

4. B. Aydemir, S. Fank, C. Vatan, Proficiency technical protocol for tensile and flexural strength, UME-KV-10-03 (TUBITAK UME, 2011)

5. EN ISO/IEC 17043, Conformity assessment - General requirements for proficiency testing, 2010

6. B. Aydemir, S. Fank, Malzeme Deneylerinde (Çekme-Ĕ̆me) Ölçüm Belirsizliğinin Hesaplanması Eğitim Dokümanı G2KV-100 (TUBITAK UME, Haziran, 2011)

7. P704, TÜRKAK Yeterlilik Deneyleri ve Laboratuvarlararası Karşilatşırma Programları Prosedürü, Rev. 3, 26.01.2006

8. B. Aydemir, S. Fank, Proficiency test report for tensile and flexural strength, UME-KV-11-01 (TUBITAK UME, 2011) 